\title{
A model of JIT make-to-stock inventory with stochastic demand
}

\author{
R Ehrhardt \\ The University of North Carolina at Greensboro, USA
}

\begin{abstract}
We consider a firm that manages its internal manufacturing operations according to a just-in-time (JIT) system but maintains an inventory of finished goods as a buffer against random demands from external customers. We formulate a model in which finished goods are replenished by a small fixed quantity each time period. In the interest of schedule stability, the size of the replenishment quantity must remain fixed for a predetermined interval of time periods. We analyse the single-interval problem in depth, showing how to compute a cost-minimising value of the replenishment quantity for a given interval length, and characterising the optimal cost, inventory levels and service as functions of the interval length and initial inventory. The model displays significant cost and service penalties for schedule stability. A dynamic version of the problem is also formulated, and shown to be convex in nature with relatively easily computed optima.
\end{abstract}

Keywords: inventory; production; stochastic processes

\section{Introduction}

Just-in-time (JIT) production systems attempt to attain the ideal of eliminating inventories of raw materials, work-inprocess, and finished goods. The general approach is simple: produce (or deliver) small batches of items in the precise amounts needed by subsequent production processes (or customers) at exactly the time needed. In general, JIT is a pull system, in which a production process pulls material from a prior process (which doesn't operate unless there is need for its output) in support of the final assembly schedule, which is closely co-ordinated with customer demand. ${ }^{1}$

There is general agreement that for JIT to work effectively, production schedules must be level and stable. ${ }^{2-4}$ While this condition is agreed upon, research has tended to focus upon translating a sequence of known future demands for products into a final assembly schedule that demands parts as uniformly as possible over time. ${ }^{5,6}$ A parallel body of research investigates methods for devising effective schedules when the production system is not organised as a JIT pull process. ${ }^{7-9}$ In both cases, the assumption of deterministic demand allows research to address the complex issues of detailed scheduling of multiple products and multiple work centres.

Research is less copious when demand is modelled as a stochastic process. Most papers model production planning problems in the manner of stochastic inventory theory, namely they regard the production process as the supplier

Correspondence: Dr R Ehrhardt, Bryan School of Business and Economics, The University of North Carolina at Greensboro, USA.

E-mail: ehrhardt@iago.uncg.edu of goods and manage the finished goods warehouse in a manner that minimises setup and inventory costs. Although many fine studies have been published, the complications of stochastic demand lead to single-item models with few details of the production process included. ${ }^{10-13}$

Most of the JIT literature, on the other hand, has been quite non-mathematical until recently. This should not be surprising, as the approach is itself inherently non-mathematical. Much of its appeal lies in its simplicity of operation and the subtle synergistic benefits that result. While promoters of JIT preach the advantages of reducing inventories to zero, some theorists and many practitioners recognise that some inventories are desirable. ${ }^{14}$ This is especially likely when certain aspects of the system are unavoidably random.

In this paper we formulate a model aimed at one of the gaps between the mathematical analysis of inventories and the practical, non-mathematical realities of JIT. A stream of research already exists for the interface between suppliers of input materials and the JIT process. ${ }^{15-17}$ Other studies have modelled both the supply and delivery ends of the process, but with deterministic demand. ${ }^{18,19}$ We seek instead to model the interface between the JIT process and the random demands of customers.

Many firms operate a JIT manufacturing process inhouse, but produce to stock, that is, they maintain an inventory of finished goods as a buffer against random customer demands. For example, consider the Toyota assembly line for Corollas, ${ }^{20}$ which produces four major model types, each type comprising about 800 unique models. The line assembles approximately 3000 cars per day according to a fixed daily schedule that includes some 
quantity of each model. The smoothed daily production schedule remains in effect for one month, and about one week before the end of the month the new daily production schedule for the upcoming month is finalised. The lead time requirement is needed to ensure that all affected parties (including suppliers and workforce schedulers) are able to make the necessary adjustments.

The modelling approach we discuss here is to minimise the expected value of costs associated with finished goods inventories while restraining changes in the replenishment quantity so as to preserve the stability of the production schedule that is so important to JIT production. Our approach, like Toyota's as described above, is to forbid changes to the replenishment quantity (or number of kanbans) for a given interval of time, and show how the optimal inventory policy and cost depend upon the length of the interval. It is then left to the manager to make an informed trade-off decision between inventory costs and schedule stability. This environment could arise whenever multiple products and capacity constraints do not allow the schedule for each product to be changed without a major effort to resolve conflicting demands for resources, forcing management to set the values of the replenishment quantities of all products simultaneously. Although the models that follow are all single-item models, they could easily form a basis for multi-item analysis.

This paper is mostly devoted to the analysis of the management of one interval of $n$ time periods during which the replenishment quantity must remain constant. The reader may wish to skip forward to the fourth section, where a dynamic model is formulated, embedding the details of this model in a macro-sequence of revision intervals. Research on the dynamic model is ongoing. The next two sections discuss $n$-period models with (1) a penalty cost for backlogged demand and (2) a service criterion that constrains the average probability of backlogging demand in any single period.

\section{Minimising expected holding and shortage costs}

We consider a single decision to fix the replenishment quantity, followed by an eventual salvage of any excess stocks or satisfaction of any backlogged demand. Consider the problem of selecting a fixed replenishment quantity $z$ to be delivered in each of $n$ consecutive periods. Let $x_{t}$ represent the inventory level at the end of period $t$, after having experienced demand for $D_{t}$ units of inventory. We assume that $\mathscr{D} \equiv\left\{D_{t}, t=1, \ldots, n\right\}$ is an i.i.d. sequence with common c.d.f. $F$ and mean $\mu$. Letting $x_{0}$ represent the beginning inventory level, we have

$$
\begin{aligned}
x_{t} & =x_{t-1}+z-D_{t} \\
& =x_{0}+t z-\sum_{i=1}^{t} D_{i}=x_{0}+t z-S_{t}, \quad t=1, \ldots, n,
\end{aligned}
$$

where the $S_{t} \equiv \sum_{i=1}^{t} D_{i}$ are the partial sums of $\mathscr{D}$. Let $F_{t}(u) \equiv P\left\{S_{t} \leqslant u\right\}$ denote the $t$-fold convolution of $F(\cdot)$.

Suppose the relevant costs consist of a linear replenishment cost and a holding or shortage cost in each period, and a salvage cost for any inventory remaining at the end of period $n$. We assume that conditions exist which make JIT production of frequent small lots attractive, namely negligible costs of changing models or products. Specifically, let $c$ represent the unit replenishment cost, $g(y, d)$ be the holding or shortage cost in any period if $y$ is the inventory available to meet demand and $d$ is the demand in that period, and $s(x)$ be the salvage value of $x$ items of inventory remaining on hand at the end of period $n$. Letting $\mathscr{C}\left(z, \mathscr{D} ; x_{0}\right)$ denote the total $n$-period cost if the initial inventory is $x_{0}$, we have

$$
\begin{aligned}
\mathscr{C}\left(z, \mathscr{D} ; x_{0}\right)= & \sum_{t=1}^{n}\left[c z+g_{t}\left(x_{t-1}+z, D_{t}\right)\right]-s\left(x_{n}\right) \\
= & n c z+\sum_{t=1}^{n} g_{t}\left(x_{0}+t z-S_{t-1}, D_{t}\right) \\
& -s\left(x_{0}+n z-S_{n}\right),
\end{aligned}
$$

where $S_{0} \equiv 0$. Suppose that $s(\cdot)$ is convex, and $g(\cdot, d)$ is convex for each $d$. Defining $\mathscr{E}$ as the expectation operator, $C\left(z ; x_{0}\right) \equiv \mathscr{E} \mathscr{C}\left(z, \mathscr{D} ; x_{0}\right)$ is a convex function, and first order conditions establish optimality.

A special case of general interest is when the salvage value, and holding/shortage cost functions are piecewise linear:

$$
\begin{gathered}
g(u, d)=h \cdot(u-d)^{+}+p \cdot(d-u)^{+} \\
s(u)=s \cdot(u)^{+}-c \cdot(-u)^{+}, \quad 0 \leqslant s \leqslant c,
\end{gathered}
$$

where $(v)^{+} \equiv \max \{v, 0\}$. That is, each excess unit costs $h$ and each unit backlogged cost $p$. At the end of period $n$ each excess unit is salvaged for a value $s$, while each unit of backlogged demand is satisfied by replenishing at cost $c$. Then

$$
\begin{aligned}
C\left(z ; x_{0}\right)= & n c z \\
& +\mathscr{E} \sum_{t=1}^{n}\left[h \cdot\left(x_{0}+t z-S_{t}\right)^{+}+p \cdot\left(S_{t}-x_{0}-t z\right)^{+}\right] \\
& -s \mathscr{E}\left(x_{0}+n z-S_{n}\right)^{+}+c \mathscr{E}\left(S_{n}-x_{0}-n z\right)^{+},
\end{aligned}
$$

which, after some manipulation, takes the form

$$
\begin{aligned}
C\left(z ; x_{0}\right)= & {\left[c\left(n \mu-x_{0}\right)+(c-s) \int_{0}^{x_{0}+n z}\left(x_{0}+n z-u\right) d F_{n}(u)\right] } \\
& +\frac{p(\mu-z) n(n+1)}{2}-n p x_{0} \\
& +(h+p) \sum_{t=1}^{n} \int_{0}^{x_{0}+t z}\left(x_{0}+t z-u\right) d F_{t}(u) .
\end{aligned}
$$


Notice that the bracketed terms represent the costs of producing and salvaging goods, while the remaining terms represent the costs of inventories and backlogs. The function is continuous and differentiable, with derivative

$$
\begin{aligned}
C^{\prime}\left(x ; x_{0}\right)= & (c-s) n F_{n}\left(x_{0}+n z\right)-\frac{p n(n+1)}{2} \\
& +(h+p) \sum_{t=1}^{n} t F_{t}\left(x_{0}+t z\right) .
\end{aligned}
$$

We consider finding the minimum value of (2) first for continuously distributed demand and then for discrete distributions.

If demand is continuously distributed, we set $C^{\prime}\left(z ; x_{0}\right)=0$, rearrange terms, and find that the optimum value of $z$ is the solution $z^{*}$ to

$$
A\left(z^{*} ; x_{0}\right)+\frac{2(c-s)}{(p+h)(n+1)} F_{n}\left(x_{0}+n z^{*}\right)=\frac{p}{(p+h)},
$$

where

$$
A\left(z ; x_{0}\right) \equiv \frac{2}{n(n+1)} \sum_{t=1}^{n} t F_{t}\left(x_{0}+t z\right)
$$

Finding $z^{*}$ using (3) is a relatively simple matter. Its left hand side involves convolutions of the demand c.d.f. and is nondecreasing in $z^{*}$. A simple linear search is all that is required.

If the demand distribution in discrete on the integers, then (2) is piecewise linear and its minimum will occur at a discontinuity in $C^{\prime}$. If $n=1$, the discontinuities in $C^{\prime}$ occur at integer values of $z$, so the minimum will occur at the smallest integer value of $z^{*}$ which makes the left side of (3) greater than or equal to the right side. This is the same as rounding up the solution of (3). If $n>1$, the discontinuities in $C^{\prime}$ occur at rational numbers, so the best integer value of $z$ may be either the rounded-up or rounded-down solution of (3). One must evaluate (2) at both possibilities and choose the value of $z$ with the smaller expected cost.

Notice that $A\left(\cdot ; x_{0}\right)$ has all the properties of a c.d.f., namely it is nondecreasing and has limits of 0 and 1 . When $n=1, A$ is the demand c.d.f., $F$. Its form is illustrated in Figure 1 for three values of $n$.

As the second term of (3) is concerned with salvage costs, it is clear that if $c=s$ the optimal order size is governed by a generalised news vendor expression, namely

$$
A\left(z^{*} ; x_{0}\right)=\frac{p}{(p+h)} \text {. }
$$

A significant consequence of this result is that, unlike the traditional newsvendor and $(s, S)$ models, minimising expected costs does not result in a target service level (probability of satisfying all demand) which is uniquely determined by the value of $p / h$. The optimal service level will generally vary from one time period to another, and the average service level

$$
\frac{1}{n} \sum_{t=1}^{n} F_{t}\left(x_{0}+t z\right)
$$

may be greater or less than the familiar ratio $p /(p+h)$ which applies to optimal newsvendor and $(s, S)$ models. ${ }^{21,22}$

The expected cost per period $C(z ; 0) / n$ is graphed in Figure 2 assuming Poisson demand with mean $4, p=9$ and $h=1$. We assume also that $c=s=0$, as this is perhaps most applicable to the long-run dynamic model for which salvage value is irrelevant and, since one typically minimises the undiscounted expected cost per period, the purchase cost is also irrelevant if unsatisfied demand is backlogged. For this case, we find that $z^{*}$ decreases from 7 to 6 to 5 as $n$

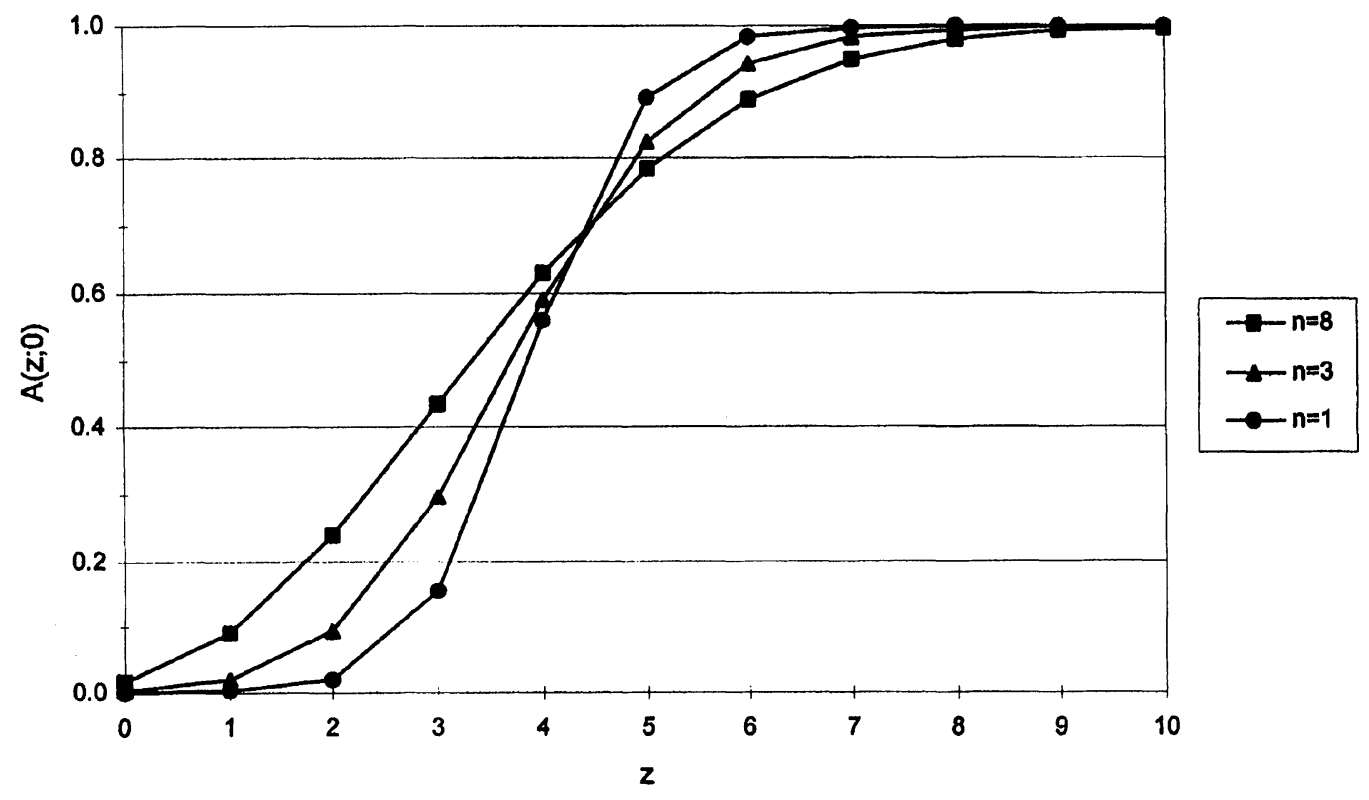

Figure $1 A(z ; 0)$ vs $z$ for $n=1,3$ and 8 ; Poisson demand with mean 4 . 


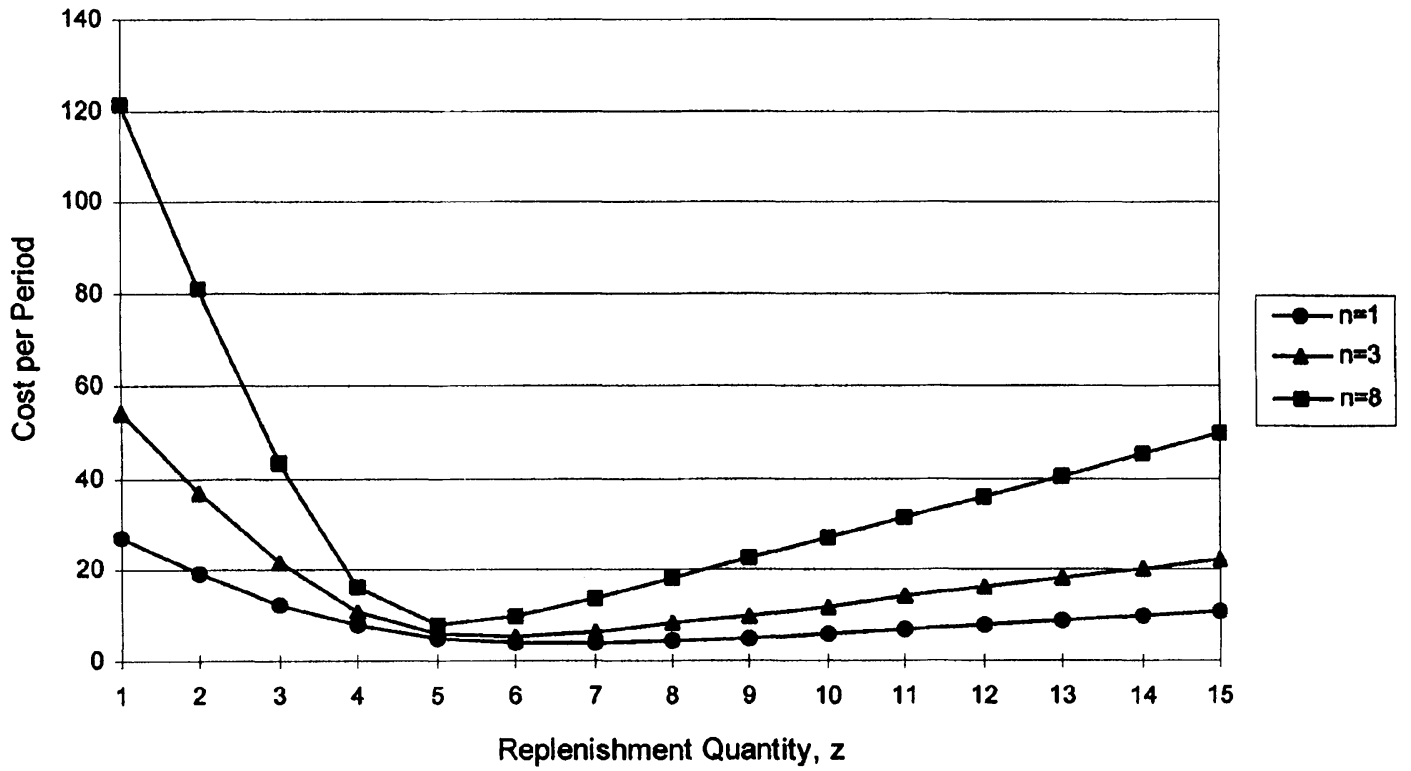

Figure 2 Average cost per period $v s z$; Poisson demand with mean $4 ; p / h=9$.

increases from 1 to 3 to 8 . Figure 2 illustrates how the penalty for choosing a non-optimal value of $z$ increases with $n$. For example, when $n=8$ the expected cost per period is nearly double the optimal value if $z$ is set at 4 rather than 5 . Here we see another significant managerial implication. The desire for a stable production schedule argues for larger values of $n$, with the unfortunate consequence of higher penalties for misspecified values of $z$. Many managers do not have precise demand information, and must rely upon statistical estimates of demand parameters, so high penalties will likely be paid for stable production schedules.

Figures 3, 4 and 5 show how the initial inventory level $x_{0}$ affects model behavior. Figure 3 demonstrates that $z^{*}$ can become less sensitive to $x_{0}$ as $n$ increases. It is clear from the form of (4) that $z^{*}$ is a non-increasing function of $x_{0}$. As $n$ increases, however, there is greater incentive to move $z$ closer to $\mu$. Figure 4 shows how the service levels of optimal policies vary by time period for three different values of $x_{0}$. When $x_{0}$ is small (or large) the replenishments are large (or small), so service tends to increase (or decrease) with time. For this particular example $x_{0}=3$ results in nearly constant service. Notice that when $x_{0}=0$ the average service level is less than the classical newsvendor optimum, ${ }^{21}$ which would be at least 0.9 . This departure from classical theory would be especially significant in situations where the unit shortage cost $p$ is difficult to measure, and management resorts to a target service level and the classical $p /(p+h)$ expression to set the value of $p$.

Figure 5 shows that in our example when $p / h$ is as high as 9 , the optimal expected inventory levels vary nearly linearly with time. Notice in particular that when $x_{0}=3$ the expected inventory increases to approximately 11 at the end of the 8-period interval, as $z^{*}=5$. Recall that Figure 3 shows a nearly constant service level for this case. The increasing variance of the convoluted demand distributions require higher inventory levels to sustain a given service level. These observations give clues to likely properties of a dynamic model which links successive multi-period intervals (see the fourth section). If service is constant during one $n$-period interval (as in Figure 4 for $x_{0}=3$ ), then it is likely that a large ending inventory level (as in Figure 5 for $x_{0}=3$ ) will cause a smaller $z$ decrease service during the next interval.

The computations described above all assume that demand follows the Poisson distribution, but they are generally representative of a wider body of results. Table 1 summarises calculations for three values of $n: 1,3$ and 8; four values of $x_{0}: 0,2,4$ and 8 ; and three families of demand distributions: $\sigma^{2} / \mu=1$ (Poisson), $\sigma^{2} / \mu=3$ (negative binomial) and $\sigma^{2} / \mu=5$ (negative binomial). Notice that $z^{*}$ and the optimal total cost per period both increase with $\sigma^{2} / \mu$ for each combination of values for $n$ and $x_{0}$. Regardless of the value of $\sigma^{2} / \mu, z^{*}$ and the optimal total cost per period display similar dependence upon the other parameters. In particular, notice that the optimal cost per period roughly doubles when $n$ is increased from 1-8 regardless of the value of $x_{0}$ or $\sigma^{2} / \mu$. Also, for each combination of values of $n$ and $\sigma^{2} / \mu, z^{*}$ increases with $x_{0}$, although the changes are smaller for larger values of $n$. Finally, focusing on each value of $x_{0}, z^{*}$ shows roughly the same dependence upon $n$ for all values of $\sigma^{2} / \mu$. When $x_{0}=0, z^{*}$ decreases with $n$; when $x_{0}=2, z^{*}$ remains constant or slightly decreases with $n$; when $x_{0}=4, z^{*}$ remains constant or slightly increases with $n$; and when $x_{0}=8, z^{*}$ increases with $n$. 


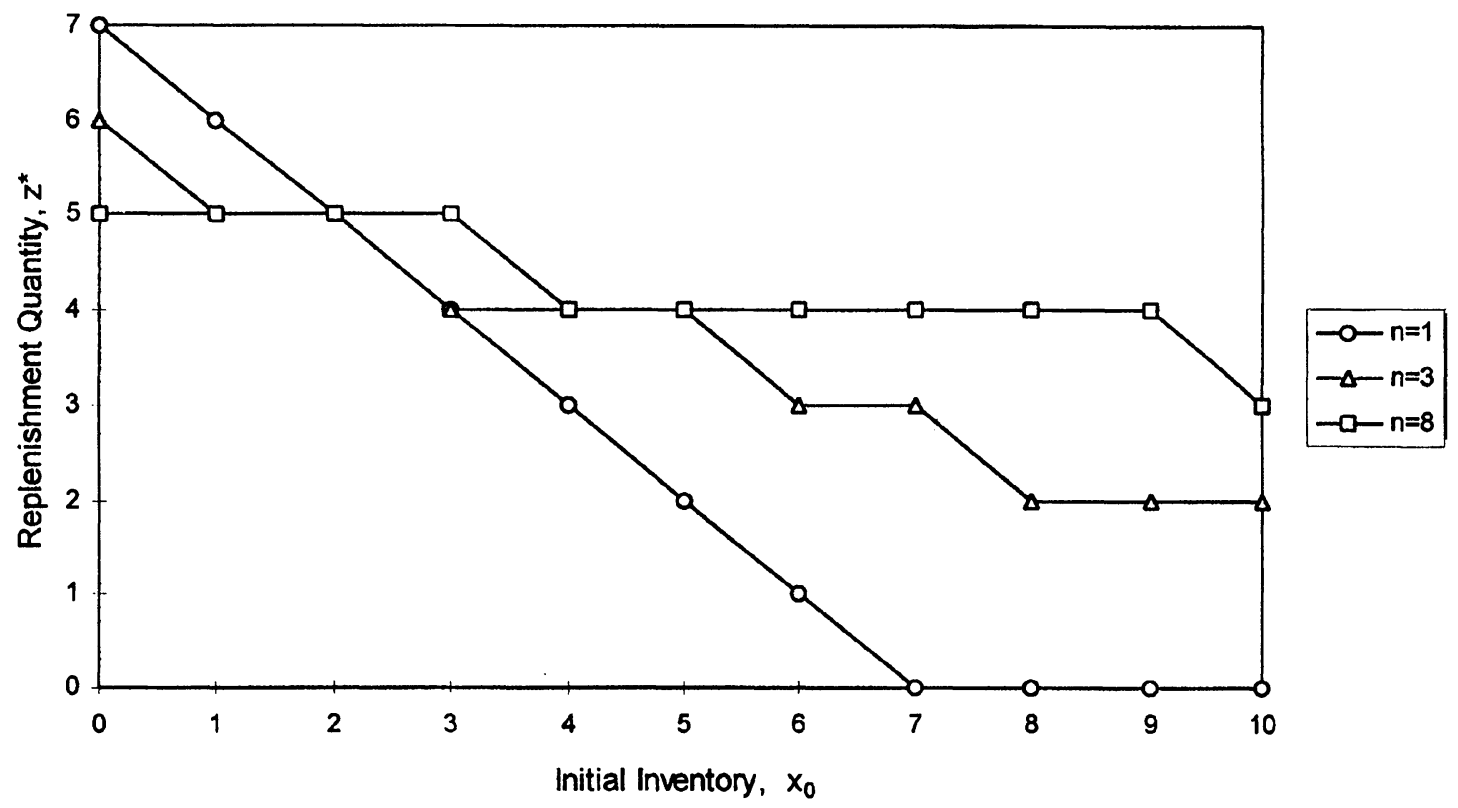

Figure 3 Optimal replenishment quantity; Poisson demand, $\mu=4, p / h=9$.

\section{Minimising inventory with a service constraint}

Many management environments do not lend themselves to the specification of a penalty cost $p$ for each unit of demand backlogged in a period. This difficulty is sidestepped in many inventory management settings by instead specifying a minimum service level and noting that a policy which minimises the expected sum of holding and shortage costs will have a service level of $p /(p+h)$ if demand is continuous (or slightly more if demand is discrete). As this result does not hold in our model, a more direct approach is of interest.
We consider a variant of the model in which we minimise the expected holding cost while constraining the average service during the $n$-period interval to be at least $\alpha$, namely

$$
\frac{1}{n} \sum_{t=1}^{n} F_{t}\left(x_{0}+t z\right) \geqslant \alpha
$$

The cost function that applies is (1) with $p=0$ :

$$
\begin{aligned}
C\left(z ; x_{0}\right)= & n c z+\mathscr{E} \sum_{t=1}^{n} h \cdot\left(x_{0}+t z-S_{t}\right)^{+} \\
& -s \mathscr{E}\left(x_{0}+n z-S_{n}\right)^{+}+c \mathscr{E}\left(S_{n}-x_{0}-n z\right)^{+},
\end{aligned}
$$

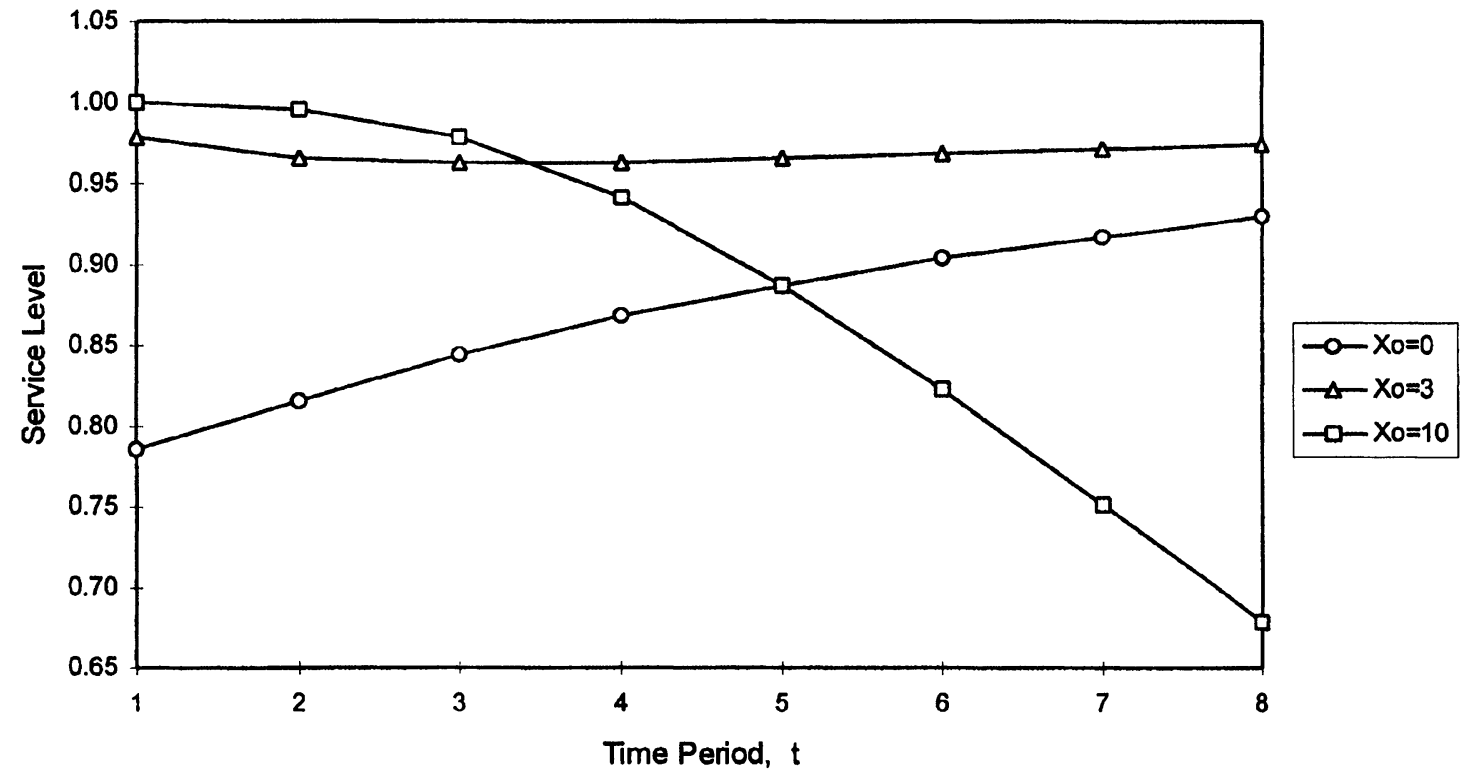

Figure 4 Optimal service levels. Poisson demand, $\mu=4, p / h=9, n=8$. 


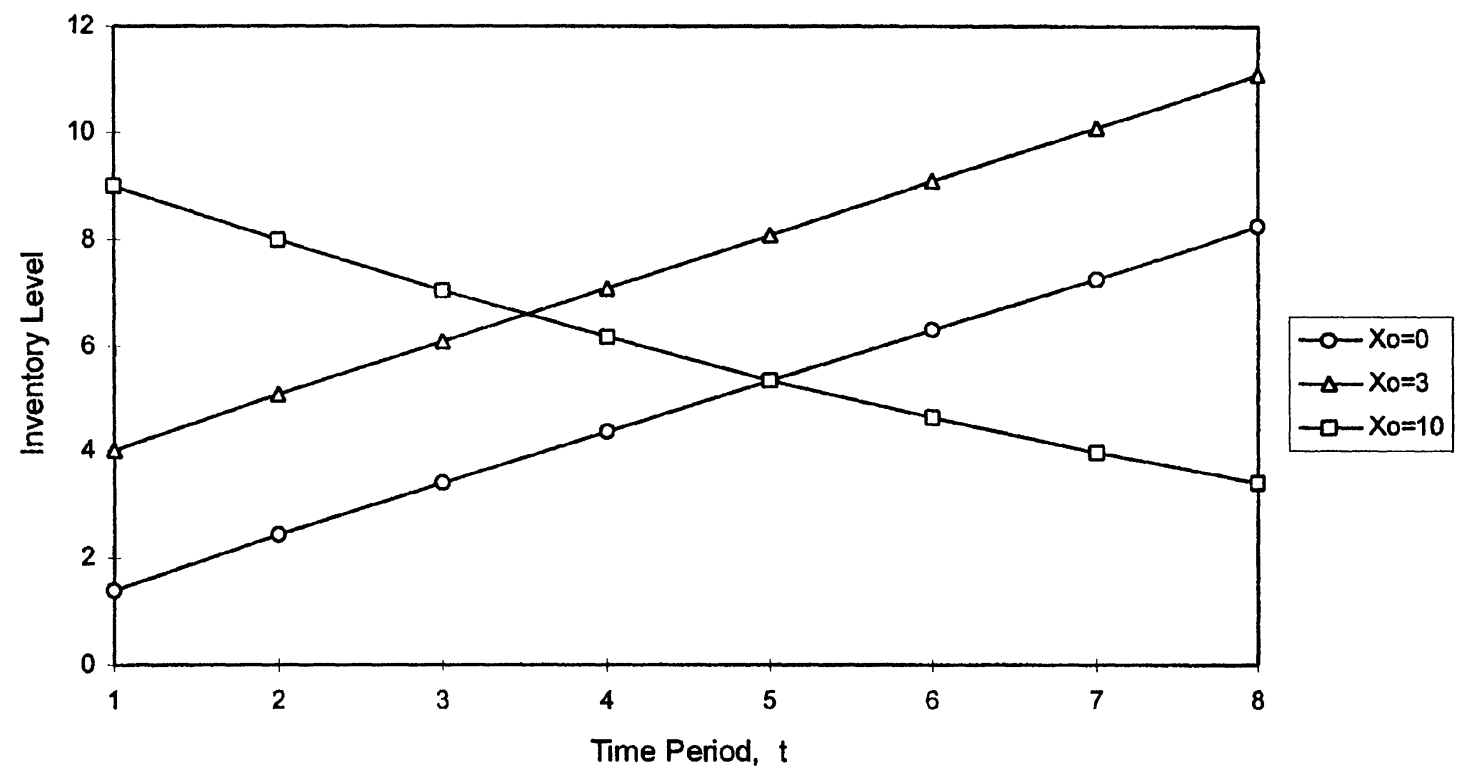

Figure 5 Expected inventory levels. Poisson demand, $\mu=4, p / h=9, n=8$.

which can be expressed as

$$
\begin{aligned}
C\left(z ; x_{0}\right)= & {\left[c\left(n \mu-x_{0}\right)+(c-s) \int_{0}^{x_{0}+n z}\left(x_{0}+n z-u\right) d F_{n}(u)\right] } \\
& +h \sum_{t=1}^{n} \int_{0}^{x_{0}+t z}\left(x_{0}+t z-u\right) d F_{t}(u) .
\end{aligned}
$$

Assuming $c \geqslant s, C\left(\cdot ; x_{0}\right)$ is nondecreasing so the optimal value of $z$ is given by the smallest nonnegative $z^{*}$ satisfying

$$
\frac{1}{n} \sum_{t=1}^{n} F_{t}\left(x_{0}+t z^{*}\right) \geqslant \alpha
$$

which is clearly a different result from what one would get using (4) with $p /(p+h)=\alpha$. Notice that (5) is also a

Table 1 Dependence of optimal policies upon $x_{0}, \sigma^{2} / \mu$ and $n$

\begin{tabular}{rrrrrrrr}
\hline & & \multicolumn{5}{c}{ Optimal policies and expected costs per period } \\
\cline { 3 - 7 }$x_{0}$ & $\sigma^{2} / \mu$ & $z^{*}$ & $C\left(z^{*} ; x_{0}\right) / n$ & $z^{*}$ & $C\left(z^{*} ; x_{0}\right) / n$ & $z^{*}$ & $C\left(z^{*} ; x_{0}\right) / n$ \\
\hline 0 & 1 & 7 & 3.85 & 6 & 5.36 & 5 & 7.91 \\
0 & 3 & 9 & 7.60 & 7 & 10.21 & 6 & 14.57 \\
0 & 5 & 10 & 10.29 & 8 & 13.74 & 7 & 19.65 \\
2 & 1 & 5 & 3.85 & 5 & 5.20 & 5 & 7.76 \\
2 & 3 & 7 & 7.60 & 6 & 10.03 & 6 & 14.50 \\
2 & 5 & 8 & 10.29 & 7 & 13.57 & 6 & 19.14 \\
4 & 1 & 3 & 3.85 & 4 & 5.37 & 4 & 8.27 \\
4 & 3 & 5 & 7.60 & 5 & 10.06 & 5 & 14.17 \\
4 & 5 & 6 & 10.29 & 6 & 13.54 & 6 & 19.00 \\
8 & 1 & 0 & 4.34 & 2 & 6.55 & 4 & 8.89 \\
8 & 3 & 1 & 7.60 & 4 & 10.52 & 5 & 14.94 \\
8 & 5 & 2 & 10.29 & 4 & 13.85 & 5 & 19.04 \\
\hline
\end{tabular}

generalised newsvendor result, as the average of convoluted c.d.f.s also satisfies all the properties of a legitimate c.d.f.

We graphically illustrate properties of this model in Figures $6-8$, assuming Poisson demand with $\mu=4$, and $\alpha=0.9$. Notice that this value of $\alpha$ is equal to $p /(p+h)$ when $p / h=9$, as we assumed in the examples of the previous section. Figure 6 shows how the optimal replenishment quantity varies with $x_{0}$ and $n$. The patterns are similar to those in Figure 3, except for higher values of $z^{*}$ in some cases. Figure 7 shows how optimal service levels increase (or decrease) with time when $x_{0}$ is small (or large). Although the patterns are somewhat different from those in Figure $4, x_{0}=3$ still results in the most nearly constant service.

In Figure $8, x_{0}=10\left(z^{*}=4\right)$ results in essentially constant inventory throughout the 8-period interval, although Figure 7 shows decreasing service for this case. When $x_{0}=0\left(z^{*}=6\right)$ or $x_{0}=3\left(z^{*}=5\right)$, however, large expected inventories are built by the end of the interval, to bring up service levels that start out low.

\section{Dynamic model formulation: sequential decisions}

Consider a sequence of $N$ decisions like the one described above in section 2. We shall refer to each sequence of $n$ periods with constant replenishment quantity as a revision interval. Let $z_{i}$ be the replenishment quantity chosen at the beginning of the $i$ th revision interval, and let $x_{i, t}$ and $D_{i, t}$ be the ending inventory and demand for the $t$ th period within the $i$ th revision interval, respectively. We assume that

$$
\mathscr{D} \equiv\left\{D_{i, t} ; t=1, \ldots, n, i=1, \ldots, N\right\}
$$




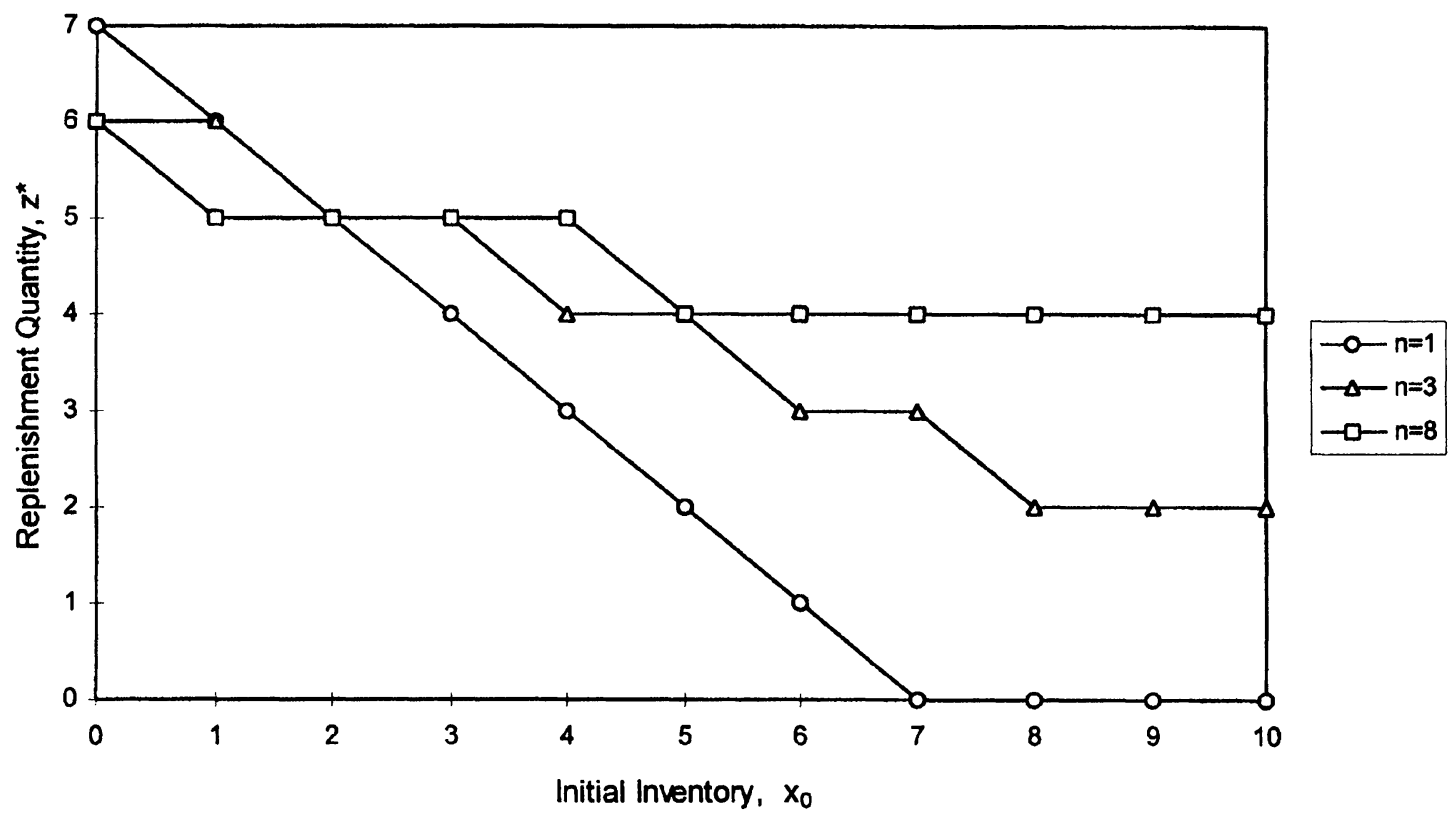

Figure 6 Optimal replenishment quantity; Poisson demand, $\mu=4, \alpha=0.9$.

is an i.i.d. sequence with common c.d.f. $F$. Letting $\mathscr{Z} \equiv\left\{z_{i}, i=1, \ldots, N\right\}$ be the sequence of replenishment quantities chosen, and $\mathscr{T}(\mathscr{Z} ; x)$ be the total cost for all revision intervals when the initial inventory is $x$, we have

$$
\begin{aligned}
\mathscr{T}\left(\mathscr{Z} ; x_{1,0}\right)= & \sum_{i=1}^{N} \sum_{t=1}^{n}\left[c z_{i}+g\left(x_{i, t-1}+z_{i}, D_{i, t}\right)\right]-s\left(x_{N, n}\right) \\
= & \sum_{i=1}^{N}\left[n c z_{i}+\sum_{t=1}^{n} g\left(x_{i, 0}+t z_{i}-S_{i, t-1}, D_{i, t}\right)\right] \\
& -s\left(x_{N, n}\right),
\end{aligned}
$$

if the replenishment quantity can be adjusted without cost at the start of each revision interval. To find the replenishment policy that minimises the expected total cost, it is convenient to formulate the problem as a dynamic program. Let

$$
G(z, x) \equiv n c z+\mathscr{E} \sum_{t=1}^{n} g\left(x+t z-S_{t-1}, D_{t}\right) .
$$

Notice that if the holding and shortage costs are linear, then $G(z, x)$ has the same form as the single decision cost function $C(z ; x)$ (1) if $s=c=0$. Defining $f_{i}(x)$ as the

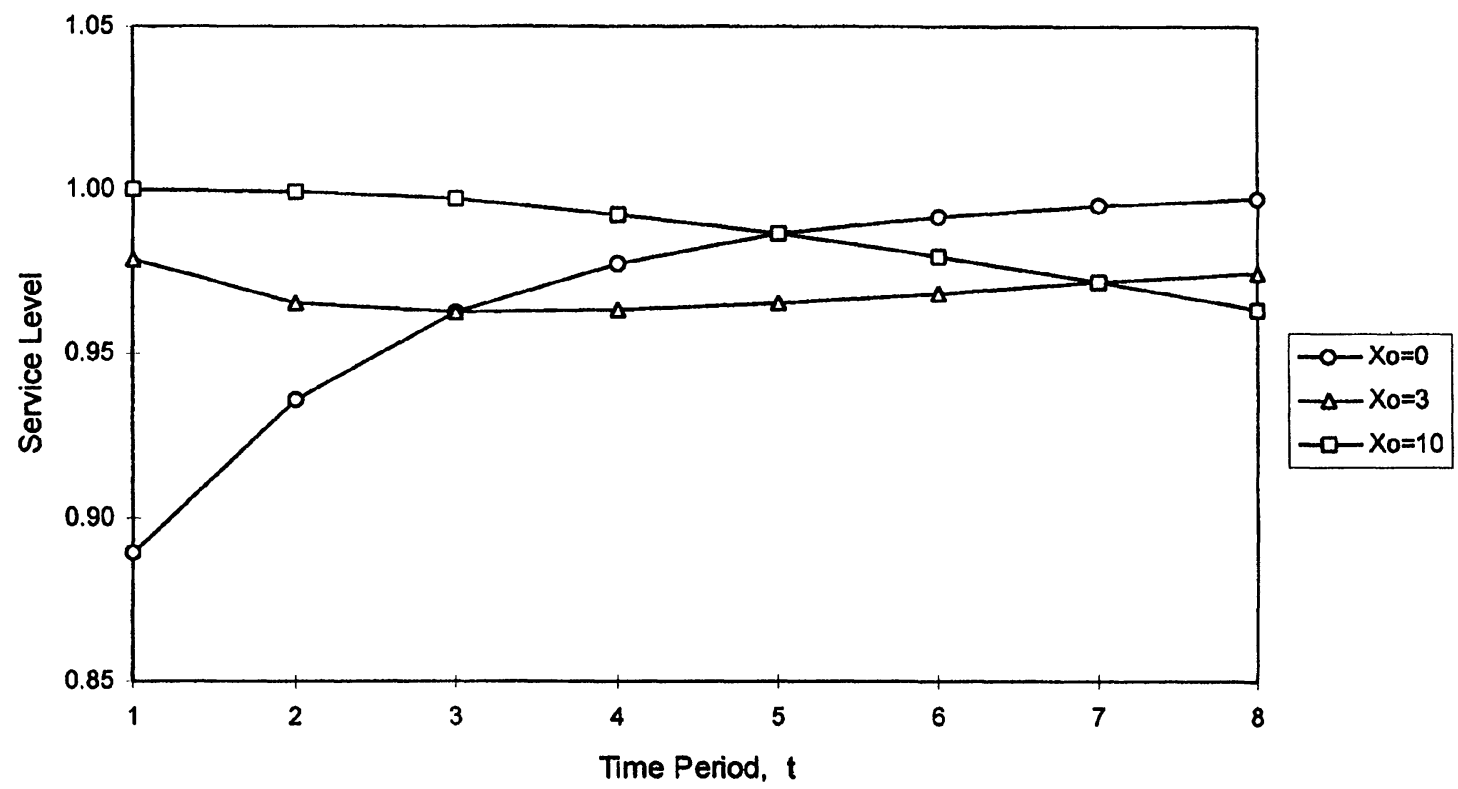

Figure 7 Optimal service levels; Poisson demand, $\mu=4, \alpha=0.9, n=8$. 


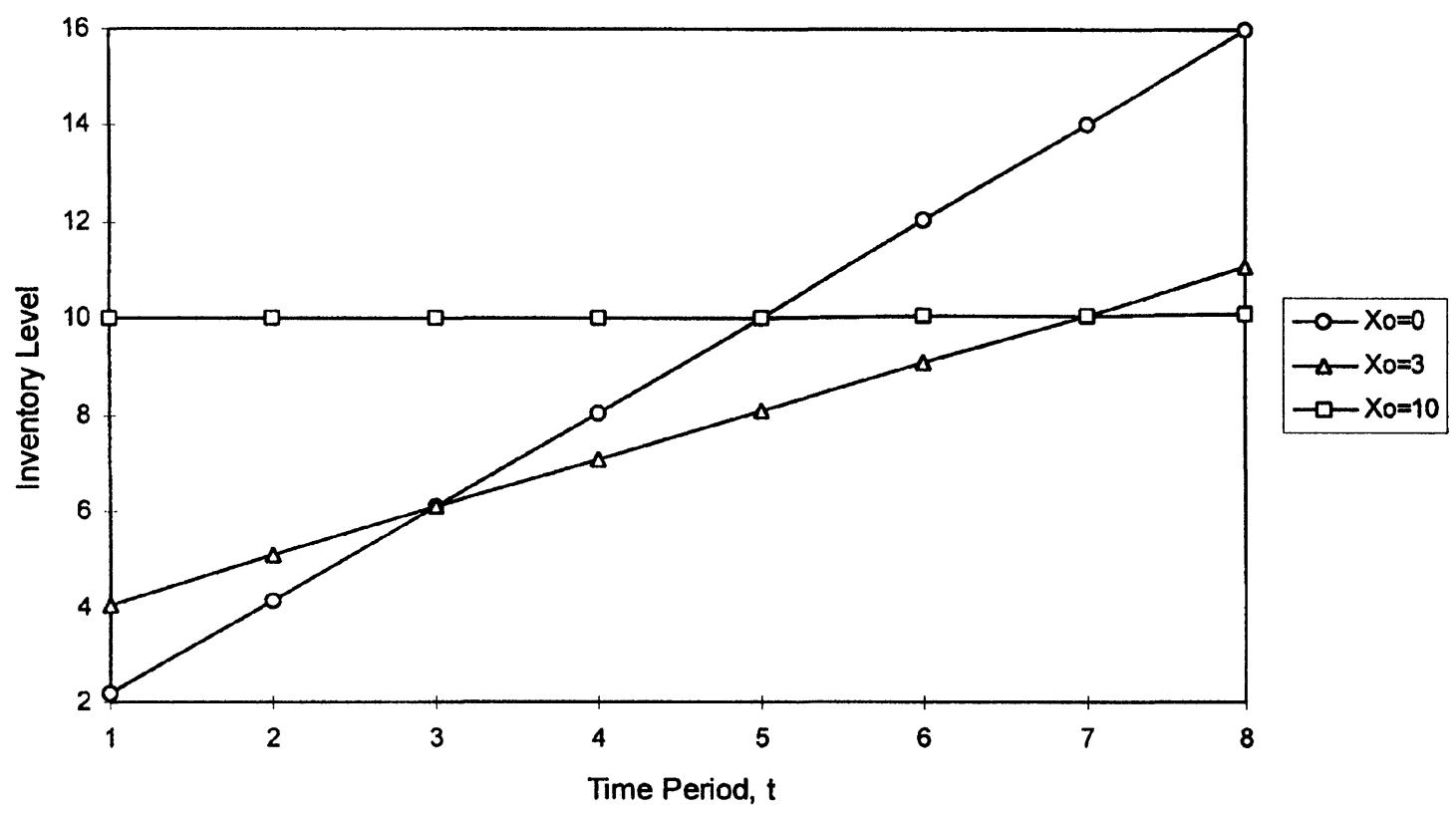

Figure 8 Optimal expected inventory levels; Poisson demand, $\mu=4, \alpha=0.9, n=8$.

minimum expected cost from revision interval $i$ through the end of the horizon, if we start revision interval $i$ with $x$ units of inventory, we have

$$
\begin{aligned}
& f_{i}(x)=\min _{z \geqslant 0}\left\{G(z, x)+\mathscr{E} f_{i+1}\left(x+n z-S_{n}\right)\right\}, i=1, \ldots, N, \\
& f_{N+1}(x)=-s(x) .
\end{aligned}
$$

The recursion is similar to those governing standard inventory problems with linear purchase cost functions. Optimal policies can be characterised by studying the properties of the function $G(z, x)$. Of particular interest is how the optimal expected cost per period varies with $n$. This information would be of managerial interest because it characterises the trade-off between the stability of the production schedule and finished goods inventory costs. The recursion is different from the standard inventory problems because the function $G$ is more complicated. It cannot be simplified by conversion to a function of $y \equiv x+z$ because of the sum over periods within each revision interval. Because of this, it almost certainly does not admit myopic optimal policies. ${ }^{23}$

Although additional research is needed to clearly understand this model, the following observations can be made at this point. First, the recursion formulated above devines a Markov decision process so we can conclude that the infinite horizon version of the problem has a stationary optimal policy. Even though the problem is more complicated than the standard stochastic inventory problem, the cost functions are all convex so that relatively simple rules should govern the computation of optimal policies. It seems reasonable to speculate that the infinite horizon optimal policy for a given value of $n$ would be given by a function $z^{*}(x)$, a non decreasing function of the starting inventory level $x$ of the revision interval. Results from the singledecision model (second section) suggest that the expected cost per period would be increasingly sensitive to misspecified replenishment quantities as $n$ increases, a property with potentially serious implications for management decision making.

Other versions of the dynamic model which could be analysed include (i) a cost associated with changing the production quantity, and (ii) the possibility of adjusting the inventory level at the same time as the production quantity. Variant (i) is quite similar to the classical production smoothing problem, which has very complex and difficult-to-compute optimal policies, while (ii) is an easier problem to analyse, but not very realistic.

\section{Summary and conclusions}

We have formulated a variety of models for studying the trade-off between finished goods inventory costs and JT manufacturing schedule stability. First we studied a single decision to fix replenishment quantities for a sequence of $n$ periods, and found a generalisation of the classical newsvendor result. Numerical illustrations suggest that as $n$ grows larger, expected costs per period become more sensitive to setting the replenishment quantity suboptimally. This could have serious implications for the manager who wishes to have a very stable production schedule and therefore desires a large value of $n$. We have also shown that the model which minimises the sum of expected holding and shortage costs does not exhibit the familiar newsvendor service level, mailing the choice of an appropriate penalty cost $p$ more difficult in some applied 
settings. Numerical illustrations also indicate that increasing inventory levels are needed to provide approximately constant service, or conversely, that decreasing service results from approximately constant expected inventory levels.

We also formulated a dynamic version of the model and showed how it is more complicated than the classical stochastic inventory model. The infinite horizon model should have a stationary optimal policy with a reasonably simple form, though. Future research will focus on the efficient computation of optimal policies, near-optimal heuristic policies, and the behaviour of the dynamic model when demand parameters must be statistically estimated from historical data.

Acknowledgements - I would like to thank the Department of Management Systems and Sciences, University of Hull, Hull, UK, for supporting this research while I was a Visiting Scholar, and the referees whose suggestions improved this paper. This work was also partially supported by NSF Grant \# DMI-9622647.

\section{References}

1 Krajewski LJ and Ritzman LP (1993). Operations Management, Strategy and Analysis. Addision-Wesley: Reading, MA.

2 Hall R (1983). Zero Inventories. Dow Jones-Irwin: Homewood, IL.

3 Monden Y (1983). Toyota Production Systems. Industrial Engineering and Management Press: Norcross, GA.

4 Vollmann TE, Berry WL and Whybark DC (1992). Manufacturing Planning and Control Systems. Business One Irwin: Homewood, IL.

5 Miltennburg J (1989). Level schedules for mixed-model assembly lines in just-in-time production systems. Mgmt Sci 35: 192207.

$\rightarrow$ Steiner G and Yeomans S (1993). Level schedules for mixedmodel, just-in-time processes. Mgmt Sci 39: 728-735.

$\rightarrow$ Jackson PL, Maxwell WL and Muckstadt JA (1988). Determining optimal reorder intervals in capacitated production-distribution systems. Mgmt Sci 34: 938-958.

$\rightarrow$ Maxwell WL and Muckstadt JA (1985). Establishing consistent and realistic reorder intervals in production-distribution systems. Ops Res 33: 1316-1341. $\rightarrow$ Roundy R (1985). 98\%-effective integer-ratio lot-sizing for onewarehouse multi-retailer systems. Mgmt Sci 31: 1416-1430.

$\rightarrow$ Gavish B and Graves SC (1980). A one-product production/ inventory problem under continuous review policy. Ops Res 28: 1228-1236.

$\rightarrow$ Doshi B, van der Duyn Schouten FA and Talman AJ (1978). A production-inventory problem with a mixture of backorders and lost sales. Mgmt Sci 24: 1078-1086.

12 De Kok AG, Tijms HC and van der Duyn Schouten FA (1984). Approximations for the single-product production-inventory problem with compound poisson demand and service level constraints. Adv Appl Prob 16: 378-401.

$\rightarrow$ Altiok T (1989). $(R, r)$ production/inventory systems. Ops Res 37: $266-276$.

$\rightarrow$ Zangwill WI (1987). From EOQ towards ZI. Mgmt Sci 33: 1209-1223.

15 Yano CA and Gerchak Y (1989). Transportation contracts and safety stocks for just-in-time deliveries. J Mfg and Ops Mgmt 2: 314-330.

16 Pan AC and Liao CJ (1989). An inventory model under just-intime purchasing agreements. Prod Inv Mgmt 30: 49-52.

17 Ramasesh RV (1990). Recasting the traditional inventory model to implement just-in-time purchasing. Prod Inv Mgmt 31: 7175.

18 Goldhar DY and Sarker BR (1992). Economic manufacturing quantity in a just-in-time delivery system. Int J Prod Res 30: 961-972.

19 Sarker BR and Parija GR (1994). An optimal batch size for a production system operating under a fixed-quantity, periodic delivery policy. $J$ Opl Res Soc 45: 891-900.

20 Webster S and Weng ZK (1995). Improving repetitive manufacturing systems: analysis and insights. Working paper, School of Business Adminstration, University of WisconsinMadison, Madison, WI 53706: 1.

21 Veinott AF Jr (1965). The status of mathematical inventory theory. Mgmt Sci 12: 745.

22 Veinott AF Jr and Wagner HM (1965). Computing optimal (s, S) inventory policies. Mgmt Sci 11: 539.

23 Heyman DP and Sobel MJ (1984). Stochastic Models in Operations Research, Vol. II. McGraw-Hill: New York, pp. 64-71. 